\title{
Loss of arginine vasopressin- and vasoactive intestinal polypeptide-containing neurons and glial cells in the suprachiasmatic nucleus of individuals with type 2 diabetes
}

\author{
Rick Hogenboom ${ }^{1,2,3} \cdot$ Martin J. Kalsbeek ${ }^{1,2,3} \cdot$ Nikita L. Korpel $^{1,2,3} \cdot$ Paul de Goede ${ }^{1,2,3} \cdot$ Marit Koenen $^{1,2,3}$. \\ Ruud M. Buijs ${ }^{4}$ - Johannes A. Romijn ${ }^{5}$ - Dick F. Swaab ${ }^{3}$. Andries Kalsbeek ${ }^{1,2,3} \cdot$ Chun-Xia Yi ${ }^{1,2,3}$ (D)
}

Received: 2 April 2019 / Accepted: 11 June 2019 / Published online: 20 July 2019

(C) The Author(s) 2019

\begin{abstract}
Aims/hypothesis The central pacemaker of the mammalian biological timing system is located within the suprachiasmatic nucleus $(\mathrm{SCN})$ in the anterior hypothalamus. Together with the peripheral clocks, this central brain clock ensures a timely, upto-date and proper behaviour for an individual throughout the day-night cycle. A mismatch between the central and peripheral clocks results in a disturbance of daily rhythms in physiology and behaviour. It is known that the number of rhythmically expressed genes is reduced in peripheral tissue of individuals with type 2 diabetes mellitus. However, it is not known whether the central SCN clock is also affected in the pathogenesis of type 2 diabetes. In the current study, we compared the profiles of the SCN neurons and glial cells between type 2 diabetic and control individuals.

Methods We collected post-mortem hypothalamic tissues from 28 type 2 diabetic individuals and 12 non-diabetic control individuals. We performed immunohistochemical analysis for three SCN neuropeptides, arginine vasopressin (AVP), vasoactive intestinal polypeptide (VIP) and neurotensin (NT), and for two proteins expressed in glial cells, ionised calcium-binding adapter molecule 1 (IBA1, a marker of microglia) and glial fibrillary acidic protein (GFAP, a marker of astroglial cells).

Results The numbers of AVP immunoreactive (AVP-ir) and VIP-ir neurons and GFAP-ir astroglial cells in the SCN of type 2 diabetic individuals were significantly decreased compared with the numbers in the SCN of the control individuals. In addition, the relative intensity of AVP immunoreactivity was reduced in the individuals with type 2 diabetes. The number of NT-ir neurons and IBA1-ir microglial cells in the SCN was similar in the two groups.

Conclusions/interpretation Our data show that type 2 diabetes differentially affects the numbers of AVP- and VIP-expressing neurons and GFAP-ir astroglial cells in the SCN, each of which could affect the daily rhythmicity of the SCN biological clock machinery. Therefore, for effectively treating type 2 diabetes, lifestyle changes and/or medication to normalise central biological clock functioning might be helpful.
\end{abstract}

Keywords Astroglialcells $\cdot$ Biologicalclock $\cdot$ Insulin resistance $\cdot$ Microglia $\cdot$ Neurotensin $\cdot$ Rhythmicity $\cdot$ Type 2 diabetes mellitus

Rick Hogenboom and Martin J. Kalsbeek contributed equally to this study.

Electronic supplementary material The online version of this article (https://doi.org/10.1007/s00125-019-4953-7) contains peer-reviewed but unedited supplementary material, which is available to authorised users.

Chun-Xia Yi

c.yi@amsterdamumc.nl

1 Department of Endocrinology and Metabolism, Amsterdam University Medical Centers (UMC), Location AMC, Meibergdreef 9, 1105 AZ Amsterdam, the Netherlands

2 Laboratory of Endocrinology, Amsterdam University Medical Centers (UMC), University of Amsterdam, Amsterdam Gastroenterology and Metabolism, Amsterdam, the Netherlands
3 Netherlands Institute for Neuroscience, an Institute of the Royal Netherlands Academy of Arts and Sciences, Amsterdam, the Netherlands

4 Department of Cell Biology and Physiology, Institute for Biomedical Research, Universidad Nacional Autonoma de Mexico, Mexico City, Mexico

5 Department of Medicine, Amsterdam University Medical Centers (UMC), University of Amsterdam, Amsterdam, the Netherlands 


\section{Research in context}

\section{What is already known about this subject?}

- Circadian misalignment impairs glucose metabolism in humans

- Biological clock machinery is impaired in the peripheral tissues of type 2 diabetic individuals

\section{What is the key question?}

- Is the central clock in the human suprachiasmatic nucleus (SCN) affected in type 2 diabetes?

\section{What are the new findings?}

- The numbers of arginine vasopressin immunoreactive (AVP-ir) neurons, vasoactive intestinal polypeptide (VIP-ir) neurons and glial fibrillary acidic protein immunoreactive (GFAP)-ir astroglial cells are significantly decreased in the SCN of individuals with type 2 diabetes

\section{How might this impact on clinical practice in the foreseeable future?}

- These novel findings indicate that the central biological clock in the human $\mathrm{SCN}$ is affected in type 2 diabetes. This will in turn lead to circadian misalignments and further impair glucose metabolism. Thus, lifestyle changes and/or medication targeting the biological clock may help to treat individuals with type 2 diabetes more effectively

$\begin{array}{ll}\text { Abbreviations } \\ \text { AVP } & \text { Arginine vasopressin } \\ \text { GFAP } & \text { Glial fibrillary acidic protein } \\ \text { IBA1 } & \text { Ionised calcium-binding adapter molecule 1 } \\ \text { NT } & \text { Neurotensin } \\ \text { SCN } & \text { Suprachiasmatic nucleus } \\ \text { VIP } & \text { Vasoactive intestinal polypeptide }\end{array}$

\section{Introduction}

In mammals, the circadian timing system plays a critical role in coordinating the daily and seasonal rhythmicity of all physiological and behavioural processes in the body. The master pacemaker of this timing system is located in the suprachiasmatic nucleus $(\mathrm{SCN})$ in the hypothalamus. Multiple types of neurons are involved in the SCN neuronal network [1]. In rodents, these mainly include the vasoactive intestinal polypeptide (VIP)-producing and the arginine vasopressin (AVP)producing neurons [1], and in addition to these, humans also possess neurotensin (NT)-containing neurons [2].

Type 2 diabetes mellitus is characterised by hyperglycaemia and insulin resistance. Glucose homeostasis and insulin sensitivity are tightly controlled by the circadian timing system, mainly through balancing sympathetic and parasympathetic outputs from the hypothalamus [3]. Previous studies have shown that impaired insulin secretion in prediabetic animal models results in decreased insulin signalling in the hypothalamus, leading to decreased inhibition of glucose production in the liver and impaired glucose uptake $[4,5]$. Evidence is accumulating for a link between circadian misalignment, for example, by sleep deprivation, and profound disruptions in blood glucose and insulin levels [6]. Thus far, few studies have investigated peripheral clock machinery in individuals with type 2 diabetes [7], and it has never been studied whether the central clock in the SCN itself is affected by type 2 diabetes. The current study aimed to profile and compare SCN neurons, especially the ones producing AVP, VIP and NT, as well as the astroglial cells (using glial fibrillary acidic protein, GFAP, as a marker) and microglia (using ionised calcium-binding adapter molecule 1, IBA1, as a marker) in control and type 2 diabetic individuals.

\section{Methods}

Donors Post-mortem hypothalamic tissues from 28 type 2 diabetic and 12 non-diabetic control individuals were obtained from the Netherlands Brain Bank, through autopsy approved by the Medical Ethic Committee of the VU Medical Center, the Netherlands. Individuals with Braak stage V-VI or clinically diagnosed severe dementia were excluded [8]. Sex, age, time/month of death were similar between groups (Electronic supplementary material [ESM] Table 1). Data on the latest post-absorptive blood glucose and $\mathrm{HbA}_{1 \mathrm{c}}$, although not complete, as indications of glycaemic control are presented in ESM Table 1. Other donor details, including post-mortem delay, clinical diagnosis, diagnosed high blood pressure, insulin treatment and cause of death are provided in ESM Table 1.

Immunohistochemistry and image analysis Immunohistochemistry for AVP-ir, VIP-ir, NT-ir, GFAP-ir and IBA1-ir cells in the SCN was performed (see ESM Methods). 
Images were analysed by the Fiji image processing program, an ImageJ distribution (Madison, WI, USA). The soma number and relative intensity of immunoreactivity for AVP-ir, VIP-ir and NT-ir neurons; the number of GFAP-ir astroglial cells and the soma number/soma size for IBA1-ir microglia (per section) were quantified by a blinded investigator (R. Hogenboom) (see ESM Methods for further details).

The numbers of AVP-ir and VIP-ir cells at each level of the SCN were plotted along the rostral-caudal axis for all control individuals. To profile the other cells, for each individual we selected consecutive sections next to the one that contained the highest number of AVP-ir cells (ESM Fig. 1).

Statistics All data are presented as means \pm SEM. Comparisons between control and type 2 diabetic individuals were analysed by Student's $t$ test. A $p$ value of $<0.05$ was considered to be significant. Daily rhythmicity and monthly variation in the number of AVP-ir, VIP-ir, NT-ir, GFAP-ir and IBA1-ir cells in the SCN was assessed using cosinor analysis SigmaPlot 14.0 software (SPSS, Chicago, IL, USA) (see ESM Methods for further details).

\section{Results}

AVP-ir neurons were mainly distributed in the dorsal SCN, while VIP-ir neurons were mainly found in the ventral and central SCN (Fig. 1, ESM Fig. 1). NT-ir neurons were visible in the dorsomedial and ventral SCN (Fig. 1). When considering all donors $(n=40)$, no correlations were found between the numbers of SCN AVP-ir, VIP-ir and NT-ir neurons and age, post-mortem delay, post-absorptive blood glucose level or $\mathrm{HbA}_{1 \mathrm{c}}$ (ESM Fig. 2). Daily rhythmicity and monthly variation in AVP-ir, VIP-ir and NT-ir neurons in the SCN did not reach significance, but acrophase and amplitude in daily rhythmicity were significant in the AVP-ir neurons in non-diabetic control individuals (ESM Figs. 3, 4). The overall numbers of AVP-ir and VIP, but not NT-ir, neurons, were significantly reduced in the SCN of type 2 diabetic individuals compared with control individuals (Fig. 1). Furthermore, compared with that in the control individuals, the relative intensity of AVP-ir was significantly lower in type 2 diabetic individuals (Fig. 1d), indicating a decrease in cellular AVP protein expression in type 2 diabetic individuals. This decrease was not observed for VIP-ir and NT-ir neurons (Fig. 1 h,l). High blood pressure was diagnosed in a large number of control donors and type 2 diabetic donors, especially those receiving insulin treatment (ESM Table 1). Previous studies have found decreased numbers of AVP-ir, VIP-ir and NT-ir neurons in individuals with hypertension [9]; however, we only found significant reductions in AVP-ir and VIP-ir neurons, but not NT-ir neurons, in individuals (non-diabetic and type 2 diabetic individuals combined) diagnosed with high blood pressure (ESM Fig. 5), indicating the reductions in AVP-ir and VIP-ir neurons might be related to insulin resistance rather than high blood pressure. Previous studies also showed a reduction in $A V P$ mRNA expression in the SCN of individuals that had
Fig. 1 AVP-ir neurons, VIP-ir neurons and NT-ir neurons in the SCN of non-diabetic and type 2 diabetic individuals.

Representative images of AVP-ir $(\mathbf{a}, \mathbf{b}), \operatorname{VIP}-i r(\mathbf{e}, \mathbf{f})$ and NT-ir (i, j) neurons in the SCN of nondiabetic (Ctrl) and type 2 diabetic (T2DM) individuals. Comparison of the number of soma of the AVP-ir (c), VIP-ir (g) and NT-ir (k) neurons, the relative intensity of immunoreactivity of the AVPir (d), VIP-ir (h) and NT-ir (I) neurons (shown as fold of Ctrl) in the SCN of non-diabetic and type 2 diabetic individuals. Data are presented as mean $\pm \mathrm{SEM}$. ${ }^{*} p<0.05, * * * p<0.001$. III, third cerebral ventricle. Scale bar, $300 \mu \mathrm{m}$
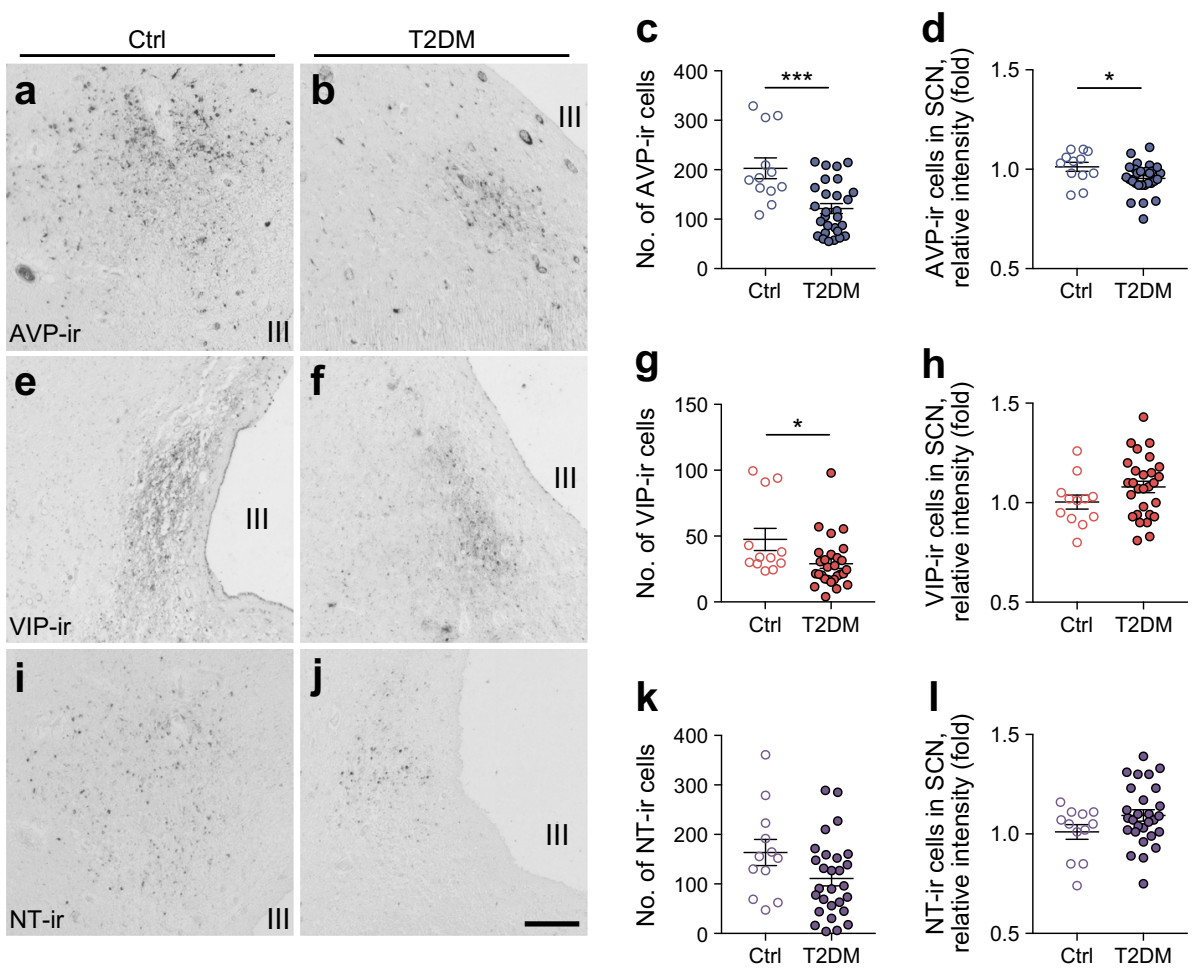
received corticosterone treatment [10]. In our study, very few individuals (one non-diabetic donor, four with type 2 diabetes) had received corticosterone treatment. We found no differences in the numbers of AVP-ir, VIP-ir and NT-ir neurons in corticosterone-treated vs non-treated individuals (ESM Fig. 6).

Overall, the number of cells showing GFAP immunoreactivity was relatively low (Fig. 2a-d) compared with the number of peptidergic SCN neurons. In some individuals, more commonly, type 2 diabetic individuals, very few GFAP-ir cells were detected in the SCN. GFAP-ir cells were only analysed by cell number. For IBA1-ir microglial cells, the number of soma and the soma size $\left(>20 \mu \mathrm{m}^{2}\right)$ were quantified (Fig. $2 \mathrm{e}-$ h). When considering all subjects together, no correlation was found between the number of GFAP-ir astroglial cells and IBA1-ir microglial cells in the SCN and age, post-mortem delay, blood glucose level or $\mathrm{HbA}_{1 \mathrm{c}}$ (ESM Fig. 2d,e). Daily rhythmicity in the number of GFAP-ir astroglial and IBA1-ir microglial cells did not reach significance (ESM Fig. 3). Interestingly, in control individuals, the amplitude of monthly fluctuation of IBA1-ir microglial cell number was more strongly statistically significant than in type 2 diabetic individuals (ESM Fig. 4). Similar to the observations for AVP-ir neurons, the number of GFAP-ir cells in the SCN was reduced in type 2 diabetic individuals (Fig. 2d). However, no correlation was found between the number of AVP-ir and GFAP-ir cells (data not shown). The total number of IBA1-ir microglial cells and their average soma size did not differ between control and type 2 diabetic individuals (Fig. $2 \mathrm{~g}, \mathrm{~h}$ ). Moreover, no difference was found in GFAP-ir astroglial and IBA1-ir microglial cells between individuals with and without high blood pressure (ESM Fig. 5). Although both astroglial and microglial cells are involved in neuroinflammation [11] and therefore could be affected by corticosterone treatment, no difference was found between corticosterone-treated and non-treated individuals in terms of numbers of GFAP-ir astroglial and IBA1-ir microglial cells (ESM Fig. 6).

\section{Discussion}

In the current study, we performed an analysis of SCN AVP-ir, VIP-ir and NT-ir neurons and IBA1-ir and GFAP-ir glial cells in post-mortem human brain tissue obtained from nondiabetic and type 2 diabetic individuals. Our analysis revealed that the numbers of AVP-ir neurons, VIP-ir neurons and GFAP-ir astroglial cells is significantly decreased in the SCN of type 2 diabetic individuals.

Some major obstacles currently hamper translational studies on brain dysfunction in type 2 diabetic individuals at the molecular level. First, there is no perfect animal model that fully mimics the pathogenesis of type 2 diabetes in humans. Second, although non-invasive brain imaging techniques have provided data on overall changes in brain metabolism in type 2 diabetes, it is poorly understood what these changes mean for specific brain regions and individual cells. In the current study, the unique collection of the Netherlands Brain Bank, with fully informative medical records, gave us the opportunity to retrogradely analyse the medical characteristics of type 2 diabetic individuals and control subjects, and systemically study differences in their brains at the molecular level.

One of the major targets of the SCN projections is the hypothalamic pre-autonomic neurons $[12,13]$. The loss of AVP-ir and VIP-ir SCN neurons, therefore, could result in a

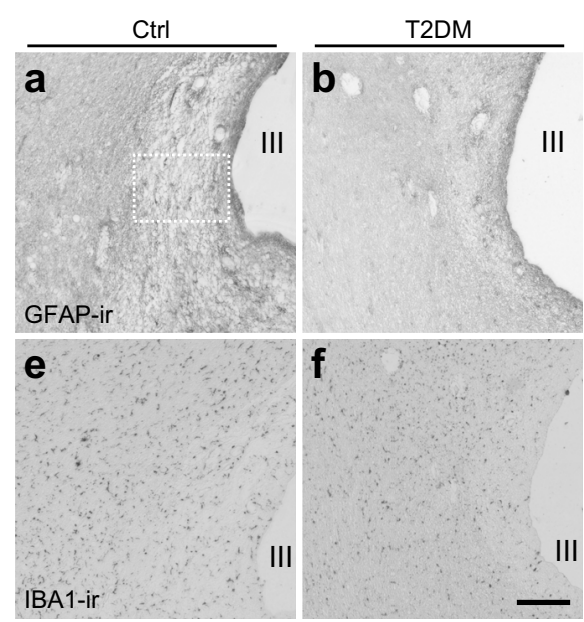

Fig. 2 GFAP-ir astroglial cells and IBA1-ir microglial cells in the SCN of non-diabetic and type 2 diabetic individuals. Representative images of GFAP-ir (a, b) and IBA1-ir (e, f) in the SCN of non-diabetic control (Ctrl) and type 2 diabetic (T2DM) individuals. (c) Higher magnification image of the area framed in (a) (arrows point to GFAP-ir cells). Comparison of
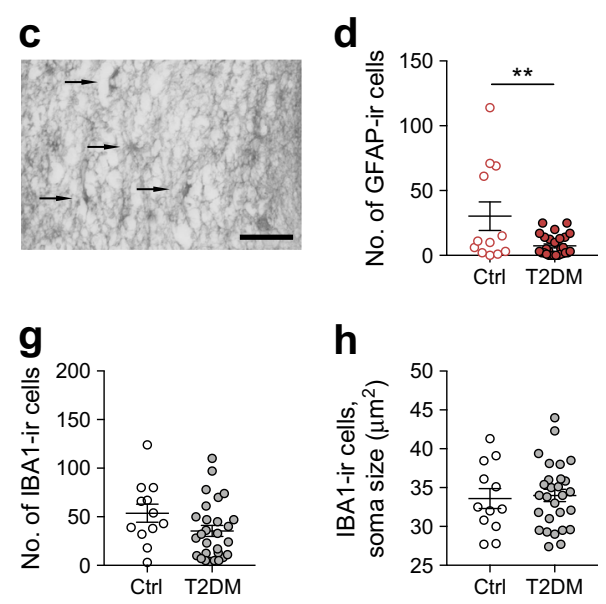

number of soma of GFAP-ir cells (d) and number of soma (g) and soma size (h) of the IBA1-ir cells in the SCN of Ctrl and type 2 diabetic individuals. Data are presented as mean \pm SEM. $* * p<0.01$. III, third cerebral ventricle. Scale bar, $300 \mu \mathrm{m}$ in $(\mathbf{a}, \mathbf{b}, \mathbf{e}, \mathbf{f}) ; 100 \mu \mathrm{m}$ in $(\mathbf{c})$ 
disbalanced autonomic hypothalamic output, as often observed in type 2 diabetes [14]. Intriguingly, the number of GFAP-ir astroglial cells was reduced in the SCN of type 2 diabetic donors, suggesting that astroglial cells play an important role in maintaining SCN function. Indeed, a recent study demonstrated that in the absence of other cellular clocks, the cell-autonomous astroglial intracellular transcription-translation negative feedback loops alone could drive molecular oscillations in the SCN and circadian behaviour in mice [15].

Previous studies have shown that individuals with type 2 diabetes have a more irregular sleep/wake cycle than the general population [16]. The 'cause-effect' question is whether the reduced number of AVP-ir and VIP-ir neurons and GFAPir astroglial cells is responsible for the disturbed sleep/wake rhythms or whether the disturbed sleep/wake rhythms affected the SCN. Observations in elderly people and ageing rats suggest the former, since increasing daytime light exposure not only improved sleep/wake rhythms but also increased AVP-ir in the SCN [17]. Nevertheless, whether modifying light exposure can improve the sleep/wake rhythm of individuals with type 2 diabetes and eventually add benefits to their treatment remains to be explored.

In summary, to start to understand the association between circadian clockwork perturbations and the metabolic syndrome in humans, we took advantage of the unique collection of type 2 diabetes human brain tissue in the Netherlands Brain Bank, and systematically analysed SCN cells. Our data indicate that besides regular glucose-lowering medication, normalising circadian rhythms by pharmacological or behavioural approaches might be helpful to treat type 2 diabetes more effectively.

Acknowledgements We thank E. Fliers and S. E. la Fleur (Department of Endocrinology and Metabolism, Amsterdam University Medical Centers [UMC], location AMC, Amsterdam, the Netherlands) for intellectual input.

Data availability All data generated or analysed during this study are included in this published article (and the accompanying ESM).

Funding This work was supported by an AMC fellowship (CXY, 2014, Amsterdam University Medical Center), the Dutch Diabetes Research Foundation (CXY, Diabetes Fonds, 2015.82.1826), and the ZonMW TOP grant (MJK, PdeG and AK, no. 91214047).

Duality of interest The authors declare that there is no duality of interest associated with this manuscript.

Contribution statement Data acquisition was performed by RH, MJK, NLK and MK. Data analysis and interpretation was performed by RH, PdG, RMB, JAR, DFS, AK and CXY. CXY and AK conceived and designed the study, RH, MJK, PdG, CXY and AK drafted the manuscript. NLK, MK, RMB, JAR and DFS revised the manuscript. All authors approved the final version of the manuscript. CXY is the guarantor of this work.
Open Access This article is distributed under the terms of the Creative Commons Attribution 4.0 International License (http:// creativecommons.org/licenses/by/4.0/), which permits unrestricted use, distribution, and reproduction in any medium, provided you give appropriate credit to the original author(s) and the source, provide a link to the Creative Commons license, and indicate if changes were made.

\section{References}

1. Antle MC, Silver R (2005) Orchestrating time: arrangements of the brain circadian clock. Trends Neurosci 28(3):145-151. https://doi. org/10.1016/j.tins.2005.01.003

2. Mai JK, Kedziora O, Teckhaus L, Sofroniew MV (1991) Evidence for subdivisions in the human suprachiasmatic nucleus. J Comp Neurol 305(3):508-525. https://doi.org/10.1002/cne.903050312

3. Kalsbeek A, Yi CX, La Fleur SE, Fliers E (2010) The hypothalamic clock and its control of glucose homeostasis. Trends Endocrinol Metab 21(7):402-410. https://doi.org/10.1016/j.tem.2010.02.005

4. Schwartz MW, Woods SC, Porte D Jr, Seeley RJ, Baskin DG (2000) Central nervous system control of food intake. Nature 404(6778):661-671. https://doi.org/10.1038/35007534

5. Buijs FN, Guzman-Ruiz M, Leon-Mercado L et al (2017) Suprachiasmatic nucleus interaction with the arcuate nucleus; essential for organizing physiological rhythms. eNeuro 4(2): ENEURO.0028-17.2017

6. Arble DM, Bass J, Behn CD et al (2015) Impact of sleep and circadian disruption on energy balance and diabetes: a summary of workshop discussions. Sleep 38(12):1849-1860. https://doi. org/10.5665/sleep.5226

7. Stenvers DJ, Scheer F, Schrauwen P, la Fleur SE, Kalsbeek A (2019) Circadian clocks and insulin resistance. Nat Rev Endocrinol 15(2):75-89. https://doi.org/10.1038/s41574-0180122-1

8. Braak H, Alafuzoff I, Arzberger T, Kretzschmar H, Del Tredici K (2006) Staging of Alzheimer disease-associated neurofibrillary pathology using paraffin sections and immunocytochemistry. Acta Neuropathol 112(4):389-404. https://doi.org/10.1007/s00401-0060127-z

9. Goncharuk VD, van Heerikhuize J, Dai JP, Swaab DF, Buijs RM (2001) Neuropeptide changes in the suprachiasmatic nucleus in primary hypertension indicate functional impairment of the biological clock. J Comp Neurol 431(3):320-330. https://doi.org/10. 1002/1096-9861(20010312)431:3<320::AID-CNE1073>3.0.CO; $2-2$

10. Liu RY, Unmehopa UA, Zhou JN, Swaab DF (2006) Glucocorticoids suppress vasopressin gene expression in human suprachiasmatic nucleus. J Steroid Biochem Mol Biol 98(4-5): 248-253. https://doi.org/10.1016/j.jsbmb.2005.10.002

11. Streit WJ, Mrak RE, Griffin WS (2004) Microglia and neuroinflammation: a pathological perspective. J Neuroinflammation 1(1):14. https://doi.org/10.1186/1742-2094-1-14

12. Gomez F, Chapleur M, Fernette B, Burlet C, Nicolas JP, Burlet A (1997) Arginine vasopressin (AVP) depletion in neurons of the suprachiasmatic nuclei affects the AVP content of the paraventricular neurons and stimulates adrenocorticotrophic hormone release. J Neurosci Res 50(4):565-574. https://doi.org/10. 1002/(SICI)1097-4547(19971115)50:4<565::AID-JNR7>3.0.CO; $2-\mathrm{C}$

13. Teclemariam-Mesbah R, Kalsbeek A, Pevet P, Buijs RM (1997) Direct vasoactive intestinal polypeptide-containing projection from the suprachiasmatic nucleus to spinal projecting hypothalamic 
paraventricular neurons. Brain Res 748(1-2):71-76. https://doi.org/ 10.1016/S0006-8993(96)01246-2

14. Vinik AI, Erbas T (2013) Diabetic autonomic neuropathy. Handb Clin Neurol 117:279-294

15. Brancaccio M, Edwards MD, Patton AP et al (2019) Cellautonomous clock of astrocytes drives circadian behavior in mammals. Science 363(6423):187-192. https://doi.org/10.1126/science. aat4104

16. Nakanishi-Minami T, Kishida K, Funahashi T, Shimomura I (2012) Sleep-wake cycle irregularities in type 2 diabetics. Diabetol Metab Syndr 4(1):18. https://doi.org/10.1186/1758-5996-4-18
17. Lucassen PJ, Hofman MA, Swaab DF (1995) Increased light intensity prevents the age related loss of vasopressin-expressing neurons in the rat suprachiasmatic nucleus. Brain Res 693(1-2):261-266. https://doi.org/10.1016/0006-8993(95)00933-H

Publisher's note Springer Nature remains neutral with regard to jurisdictional claims in published maps and institutional affiliations. 